\title{
Actitudes hacia el hecho multicultural
}

\author{
PEDRO ORTEGA RUIZ \\ RAMÓN MÍNGUEZ VALLEJOS \\ Universidad de Murcia
}

\begin{abstract}
SUMMARY.-Attitudes towards multiculturalism
In this paper, the necessity for a multicultural education responding to the reality of plural society like ours becomes apparent. The conceptual and methodological difficulties surrounding the research into the field of multicultural education are also discussed. Finally, a scale of attitudes towards multiculturalism and a preliminar analysis of the results obtained after the students of the Teachers' Training University School were given the test are presented.
\end{abstract}

\section{NECESIDAD DE UN ENFOQUE MULTICULTURAL EN LA EDUCACIÓN}

Una de las características que mejor define a la sociedad actual es su carácter plural en valores e ideologías, propiciado, sin duda, por la abundancia de imágenes e informaciones, la disponibilidad asombrosa de medios de comunicación y la introducción de nuevas tecnologías en todos los sectores de la actividad humana, que ha posibilitado el reconocimiento de las diversas identidades culturales como un valor que ha de ser respetado y promovido.

Sin embargo, estos mismos medios han convertido el mundo en la «aldea global», en expresión de McLuhan, con un poder fantástico de convergencia y homogeneidad que ponen en peligro los rasgos propios y peculiares de los individuos y de los pueblos; la identidad cultural de éstos, en cuanto sentimiento de pertenencia que vincula al individuo a un patrimonio socio-cultural concreto; las raíces comunitarias que alimentan su «manera de estar en el mundo» (Galino, 1990). Esta nueva «era planetaria», a cuyos primeros ensayos estamos asistiendo (derrumbamiento político-ideológico y económico de los países del llamado «socialismo real»; Unidad Económica y Política de Europa; Casa Común Europea, etc.) puede suponer un encuentro entre pueblos de identidades culturales diferentes, más que la mera coexistencia de universos o mundo separados; pero también puede significar la pérdida de lo original y específico de cada pueblo, la desaparición de la «complejidad y diversidad» en aras de la uniformidad y homogeneidad cultural de los pueblos.

El reconocimiento del hecho plural de nuestra sociedad por una parte, y sus tendencias homogeneizadoras por otra, obliga a una profunda reflexión sobre la praxis educativa y los fines mismos de la institución escolar. Las diferencias en tradicio- 
nes, culturales, lenguas, valores; en otras palabras, el reconocimiento de las identidades culturales determina una diversificación del sistema educativo que debe encontrarse reflejada en formas también «diferenciadas» de enseñanza. Constituye, por otra parte, una exigencia para una enseñanza de calidad y una adaptación necesaria a las características nuevas de una sociedad cambiada (Mallick, 1982). Es del todo necesario empezar a creer y actuar sobre la idea de que las mejores escuelas no son aquellas que tienen alumnos con rendimientos académicos más altos, sino las que son más capaces de favorecer el desarrollo de todos los alumnos de acuerdo con sus posibilidades (Marchesi, 1990) en función del contexto sociocultural al que pertenecen.

No es objeto de este trabajo establecer cuáles son las diferencias culturales que podrían considerarse determinantes para una educación diferenciada en base a la cultura. Algunos autores (Pratte, 1981) señalan la necesidad del carácter fundamental de las mismas; es decir, sólo cuando dichas diferencias sean capaces de generar en el individuo un conjunto de valores y actitudes que configuren en el mismo un modo «diferenciado» de afrontar la vida. Entre tales diferencias habría que considerar la lengua, las características étnicas y los «modos propios» de hacer de una comunidad (García Garrido, 1990).

Una de las mayores dificultades planteadas en la investigación sobre educación multicultural se centra en su falta de claridad conceptual: «serían pocos (los profesores) los que coincidieran en el significado y en la práctica de los estudios multiculturales» (Verma, 1984) porque los conceptos mismos de multiculturalismo-pluralismo cultural y de cultura con frecuencia se presentan insuficientemente delimitados (Ibáñez-Martín, 1983; Sachs, 1989).

La necesidad de un enfoque multicultural en educación no se ha traducido en investigaciones o programas educativos de contenido cultural plural, como el mismo Verma reconoce. Por el contrario, durante un cierto tiempo la educación multicultural se entendió como respuesta a un «problema étnico» planteado, marginando otros aspectos como la herencia cultural, valores, estilos de vida, etc. Su finalidad no era otra que la «asimilación o integración» de los grupos étnicos minoritarios, mediante programas de compensación, en una sociedad pretendidamente homogénea. El enfoque actual subraya más los aspectos culturales que los propiamente étnicos, ayudando a definir la propia identidad cultural, a disminuir los prejuicios y la discriminación y a promover el desarrollo de actitudes de tolerancia y de cooperación entre los individuos y grupos de diferentes culturas, a asumir la originalidad de cada pueblo (Stenhouse, 1982; UNESCO, 1990). La tendencia actual en educación multicultural, más que propugnar una educación para grupos aislados en base a su diferente identidad cultural que asegure su pervivencia o continuidad, defiende, por el contrario, una educación de todos en las diferencias culturales sobre una base común de valores compartidos (Camilleri, 1985), o compartibles por toda la sociedad. Se trata de una educación intercultural que afronte no sólo el encuentro entre culturas diversas, sino subraye, además, el carácter recíproco que el encuentro entre ellas debería suponer (Galino, 1990).

A las dificultades existentes, a nivel conceptual, en la investigación sobre educación multicultural se añaden otras a nivel operativo. No sólo falta claridad en las definiciones, sino que los objetivos de ésta con frecuencia aparecen confusos, lo que 
hace difícil que pueda darse un modelo de educación multicultural aceptable para todos (Verma, 1984). En la práctica resulta problemático determinar cuál debe ser el contenido de la educación en una sociedad plural. Son muchos los autores que hablan de la necesidad de proponer explícitamente, desde el sistema educativo, la adquisición de un conjunto de valores morales, estéticos, éticos y, en su caso, religiosos aún dentro de una sociedad plural (Ibáñez Martín, 1983; Marín Ibáñez, 1989; Singh, 1989; Gates, B. 1990). Aparte de que es imposible que pueda darse un proceso educativo sin referencia a un sistema de valores (Escámez, 1986), no deja de ser una ilusión, por ahora, poner de acuerdo a pedagogos y políticos en la construcción de un programa mínimamente común para todos los miembros de una sociedad plural que evite por una parte, su desintegración y, por otra, la asimilación y pérdida de las subculturas que la integran (Vázquez, 1989). Es posible, no obstante, desde la antropología, establecer un conjunto de valores comunes compartidos por los individuos en una sociedad plural. En la actualidad, puede considerarse como un valor unánimemente compartido el educar no sólo para unos determinados roles productivos, sino también para la tolerancia, la convivencia pacífica y democrática, la participación ciudadana, el respeto a los derechos humanos y el reconocimiento de la igualdad entre las personas, la salud y la protección del medio ambiente, el ocio y la cultura (Diseño Curricular Base. MEC, 1989).

A nuestro juicio, la presencia operativa de los valores o metas comunes de una sociedad plural en el proceso de educación no solamente es deseable, como se pregunta el prof. Vázquez (1983), sino que también es exigible. Pero ello no garantiza, por sí sólo, que estemos ante una educación pluri o multicultural que contemple las «especificidades» $o$ identidades culturales de cada grupo. Esta requiere que, sobre una base común, se de una educación de todos en las diferencias culturales, incluidas las no minoritarias, en una reciprocidad de perspectivas. Por ello es más correcto hablar de educación intercultural que ofreciese, además, una información tanto descriptiva como genética y explicativa de las diferentes culturas que integran la sociedad (Camilleri, 1985). La «unidad cultural» de un pueblo no puede constituir la finalidad de la educación, como parece sugerir Brezinka (1990), sino la libre expresión, defensa y promoción de las diversas culturas que lo integran, sobre la base común de unos valores compartidos. En todo caso, los nuevos objetivos o finalidades de la educación, en una sociedad tan plural como la nuestra, se deben establecer necesariamente a partir de una reflexión sobre el hombre concreto, aquí y ahora, reconociendo las diferencias culturales y la singularidad de cada persona en su dimensión biológica y espiritual (Diz Hochleitner, 1986; 1990). Tal proyecto educativo aspira a la generación de formas originales nacidas de las culturas que se han puesto en contacto sin dejarse reducir a ninguna de ellas, posibilitando de este modo la aparición de formas nuevas de expresión de la nueva cultura, enriquecedoras de la cultura nacional originaria (Galino, 1990).

A las dificultades antes aludidas que afectan a la investigación sobre educación multicultural podría añadirse otra de carácter metodológico: no sólo no existe acuerdo sobre qué sea educación pluri/multi/o intercultural, ni qué contenidos enseñar; tampoco disponemos de instrumentos válidos de evaluación de programas pedagógicos, en nuestro ámbito, sin los cuales, obviamente, se hace imposible cualquier investigación al respecto. A nuestro juicio, la educación intercultural, como preferi- 
mos denominarla, es también una educación en actitudes. Implica educar a los sujetos en la tolerancia, respeto y reconocimiento de las identidades culturales de los individuos y los pueblos, que se manifiestan en tradiciones distintas, costumbres, lengua, estilos o modos diferentes de vida, etc. Supone cambiar las actitudes de rechazo e indiferencia de los sujetos generadas, en gran parte, por el desconocimiento $\mathrm{y}$, en menor medida, por informaciones interesadas, filtradas a través de los distintos medios de comunicación. Los primeros resultados de nuestra investigación en curso, apuntan en esta dirección.

La escalas de actitudes están condicionadas, en su aplicación, por el contexto socio-cultural en que han sido elaboradas. Esto nos ha obligado a desechar otras escalas sobre actitudes hacia el hecho multicultural construidas en otros contextos (anglo-sajón), pero difícilmente aplicables al nuestro.

\section{CUESTIONARIO-ESCALA DE ACTITUDES HACIA EL HECHO MULTICULTURAL}

Como paso previo a sucesivas investigaciones pedagógicas, hemos construido una escala de actitudes hacia el hecho multicultural (anexo I). La misma se ha elaborado sobre la totalidad (1490) de alumnos de la E.U. del Profesorado de Murcia y una muestra de 284 , estadísticamente representativa a un nivel de confianza de $95,5 \%$ y un margen de error de $\pm 5,3 \%$.

La escala consta de trece items o proposiciones y su coeficiente de fiabilidad, en cuanto a su consistencia interna, es del 0.77 (Spearman-Brown y Guttman). Su índice de correlación biserial (R) se sitúa por encima del 0.40 en todos los items (anexo II).

Dicha escala está configurada en torno a tres factores o dimensiones en la actitud hacia el hecho multicultural. A saber: Factor I: «Pluralidad de culturas». Este factor engloba aquellos items que hacen referencia a la pluralidad de culturas existentes en el Estado, cuya manifestación más visible es la diversidad de lenguas. Factor II: «Uniformidad». Engloba los items que hacen referencia a una visión unitaria del Estado tanto en su organización administrativa como en su cultura. Factor III: «Promoción-Defensa». Engloba los items que hacen referencia a la promoción y defensa de las minorías étnicas y de la identidad cultural de cada individuo y pueblo o comunidad.

A nuestro juicio esta escala podría utilizarse como instrumento de medida de la actitud hacia el hecho multicultural, preferentemente, en aquellas comunidades del Estado que no sean bilingües. Podría ser también útil su aplicación en los alumnos de los últimos cursos de BUP-FP y COU.

\section{ANÁLISIS DE LOS DATOS ${ }^{1}$}

El análisis de los datos obtenidos nos revela que el 54,4\% de los alumnos encuestados muestra actitudes positivas hacia el factor I «Pluralidad de culturas», en

1. El programa utilizado para el tratamiento de los datos ha sido la versión 4.0 del SYSTAT, En WILKInson, L. (1988): SYSTAT: The system for statistics. Evanston, IL; Systat, Inc. 
su conjunto, cuya manifestación más visible es la lengua propia. El $28,6 \%$ considera como algo negativo el hecho multicultural y un $16,8 \%$ se muestra indiferente.

Si bien es cierto que la mayoría de los encuestados $(54,4 \%)$ muestra una actitud positiva hacia el hecho multicultural, un análisis más detallado nos revela que un $36,2 \%$ declara que es ilusorio conseguir una convivencia pacífica basada en el respeto a todas las culturas existentes en el Estado, mientras que el 11,2\% se muestra indiferente al respecto. Nos parece éste un elevado porcentaje de aquéllos que desconfían en la educación, entre otros factores, como elemento facilitador de una mejor convivencia y de respeto a las diferentes culturas. Así mismo es preocupante el elevado porcentaje ( $40 \%)$ de futuros educadores para quienes la promoción de aspectos diferenciadores-singulares de una comunidad, como es la lengua propia, contribuya a la desintegración del Estado, o se declaran indiferentes $(18,6 \%)$. Del mismo modo, un 31,6\% de los encuestados ve la diversidad de culturas existente en el Estado como una dificultad para la convivencia entre todos; diversidad o patrimonio cultural que no es necesario ni conservar ni promover.

Respecto al factor III «Promoción y defensa» de las minorías étnicas y de la identidad cultural de los individuos y pueblos el 50,3\% muestra, globalmente, actitudes favorables. Por contra, el 33,9\% lo considera negativo, y el 15,6\% es indiferente.

Sin embargo, un análisis más detallado de los items que configuran este factor nos manifiesta que el $86,6 \%$ considera un lujo innecesario y un gasto superfluo la promoción de las tradiciones, costumbres y folklore, etc. de las comunidades que integran el Estado, y un $27 \%$ no considera como un derecho fundamental el ser educado en la propia lengua. Del mismo modo, un $35,8 \%$ no considera necesaria la protección de las minorías étnicas en su lengua y en su cultura, como medio de conservar su identidad cultural; y un 37,8\% estima que la libre expresión de la cultura de un pueblo no constituye la única vía de supervivencia del mismo.

En cuanto al factor II, «Uniformidad», de nuestra escala, los datos globales obtenidos señalan una clara actitud de rechazo por parte de los encuestados hacia manifestaciones uniformes de expresión cultural como la lengua única $(85,2 \%)$ o la promoción de un Estado uniforme en su cultura y organización administrativa $(67,2 \%)$. No obstante, hay que subrayar que un $32,7 \%$ de la muestra manifiesta actitudes positivas hacia la promoción de una imagen de Estado uniforme en su cultura y centralista en la administración.

Al tratarse de futuros educadores, por tanto con una clara incidencia en el proceso educativo de los niños y adolescentes, el porcentaje de los que muestran actitudes negativas hacia el hecho multicultural, o se declaran indiferentes, lo consideramos de singular relevancia. Este porcentaje de encuestados que manifiesta estas actitudes debería dar lugar a una orientación distinta en el currículum de formación inicial del profesorado y la incorporación de nuevos métodos de enseñanza que posibiliten a los futuros educadores la elaboración de programas que contemplen la educación en actitudes positivas hacia el hecho multicultural. 


\section{BIBLIOGRAFÍA}

BREZINKA, W. (1990): La educación en una sociedad en crisis. Narcea, Madrid.

CAMILLERI, C. (1985): Antropología cultural y educación. UNESCO. París.

DíEZ HOCHLEITNER, R. (1986): Documento de trabajo básico. En: Los objetivos de la educación. Documentos de un debate. Fundación Santillana. Madrid.

DíEz HochleITNER, R. (1990): Documento de trabajo básico. En: La educación. Documentos de un debate. Fundación Santillana. Madrid.

ESCAMEZ, J. (1986): Los valores en la pedagogía de la intervención. En: Conceptos y Propuestas (III). Papers d'Educació. Nau llibres. Valencia.

GALINO, A. (1990): La educación intercultural. Orígenes y enfoques. En GALINO, A. y ESCRIBANO, A.: La educación intercultural en el enfoque y desarrollo del curriculum. Narcea. Madrid.

GARCÍA GARRIDO, J. L. (1990): Las políticas educativas en transición. En: La educación. Documentos de un debate. Ob. cit.

GATES, B. (1990): Religion, Morality and education constitutionally incongruent? Journal of Moral Education. Vol. 19, № 3, oct. pp. 147-158.

IBÁÑEZ-MARTÍN, J.A. (1983): Ambiciones y aporías del pluralismo, Rev. Española de Pedagogía. XLI. № 161 julio-sept. pp. 371-397.

IBÁÑEZ MARTÍN, J.A. (1983): La educación moral en una sociedad pluralista. La escuela en acción. Enero, pp. 17-19.

MALLICK, K. y otros (1982): Self-concept, achievement and multicultural education. MacMillan. London.

MARCHESI, A. (1990): Reforma y calidad de la educación. En: La educación. Documentos de un debate. Ob. cit.

MARÍN IBÁÑEZ, R. (1989): Valores y fines. En: AA.VV.; Filosofía de la Educación hoy. Conceptos, autores, temas. Dykinson, Madrid.

MINISTERIO DE EDUCACIÓN Y CIENCIA (1989): Diseño curricular Base. Educación Infantil. Madrid.

PRATTE, R. (1981): Diversidad cultural y educación. En: STRIKE, K. y EGAN, K. (eds): Etica y política educativa. Narcea, Madrid.

SACHS, J. (1989): Match o mismatch: Teachers' Conceptions of culture and multicultural education policy. Australian Journal of Education. Vol. 33, № 1, pp. 19-33.

SINGH, B. (1989): Neutrality and Commitment in Teaching Moral and Social Issues in a Multicultural Society. Educational Review. Vol. 41, № 3, pp. 227-242.

STENHOUSE, L. y otros (1982): Teaching about race relations: problems and effects. Routledge and Kegan Paul. London.

UNESCO (1990): Futuro de la educación. Hacia el año 2000. Narcea. Madrid.

VÁZQUEZ, G. (1989): Problemática pedagógica de la relación entre educación y pluralismo. Rev. Española de Pedagogía. 161, pp. 399-417.

VÁZQUEZ, G. (1989): El concepto de educación multicultural. En: La educación multicultural en el Estado de las Autonomías. VIII Seminario Nacional de Teoría de la Educación. San Sebastián.

VERMA, G. (1984): Educación multicultural: problemas de la investigación. En: HUSEN, T. y OPPER, S.: Educación multicultural y multilingüe. Narcea, Madrid. 


\section{Anexo I}

\section{CUESTIONARIO-ESCALA DE ACTITUDES HACIA EL HECHO MULTICULTURAL}

A continuación te planteamos una serie de cuestiones que esperamos respondas con la máxima sinceridad y libertad. Con ello pretendemos investigar algunos aspectos de la realidad social de nuestro país que podrían ser abordados convenientemente en el ámbito educativo.

Para ello te pedimos que señales tus posiciones o actitudes personales respecto a las cuestiones propuestas rodeando con un círculo la posición que consideres más aceptable para tí. Queda garantizado el anonimato de tus informaciones.

Para ayudarte en tus respuestas, transcribimos el posible sentido de las mismas:

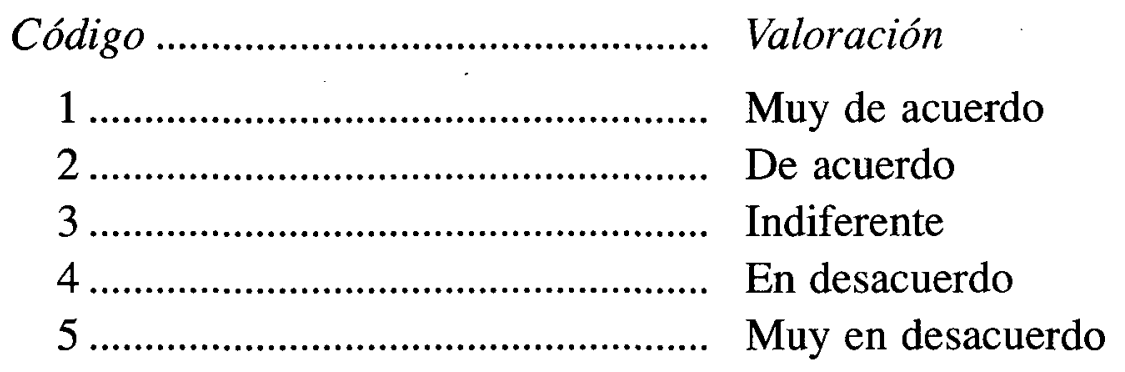

Te agradecemos muy sinceramente la colaboración que nos prestas.

1. La promoción de las propias lenguas (gallego, catalán, euskera, valenciano), en sus respectivos territorios, por parte de algunos comunidades del Estado Español, la considero:
Positiva 1
2

3
4
$5 \quad$ Negativa

2. Considero que la afirmación de la propia identidad cultural lleva consigo la infravaloración de las demás.

$\begin{array}{lllllll}\text { De acuerdo } & 1 & 2 & 3 & 4 & 5 & \text { En desacuerdo }\end{array}$

3. La utilización del castellano, como única lengua oficial en todo el territorio del Estado Español, la considero:

$\begin{array}{lllllll}\text { Positiva } & 1 & 2 & 3 & 4 & 5 & \text { Negativa }\end{array}$

4. Estimo que la denominada «pluralidad de nacionalidades y culturas» que constituyen el Estado Español es algo inventado que no responde a la realidad de los hechos.

$\begin{array}{lllllll}\text { De acuerdo } & 1 & 2 & 3 & 4 & 5 & \text { En desacuerdo }\end{array}$

5. Considero necesaria la protección de todas las minorías del Estado Español (gitanos, negros, inmigrantes, etc.) en su lengua y en su cultura como medio de conservar la identidad cultural de cada una de ellas.

$\begin{array}{lllllll}\text { De acuerdo } & 1 & 2 & 3 & 4 & 5 & \text { En desacuerdo }\end{array}$ 
6. La expresión utilizada por algunos políticos para referirse a algunas comunidades como naciones dentro del Estado Español, me resulta:
Agradable
2
3
4
5 Desagradable

7. Estimo que la consecución de la convivencia pacífica y el respeto entre todas las culturas del Estado Español es algo ilusorio.
De acuerdo
12
3
45
En desacuerdo

8. La promoción de los aspectos diferenciadores-singulares de una comunidad del Estado Español, como es la lengua propia, contribuye a la desintegración del Estado.

$\begin{array}{lllllll}\text { De acuerdo } & 1 & 2 & 3 & 4 & 5 & \text { En desacuerdo }\end{array}$

9. La promoción de la imagen de un Estado uniforme en su lengua, en su cultura y en su forma de organización político-administrativa, la considero:

$\begin{array}{llllllll}\text { Positiva } & 1 & 2 & 3 & 4 & 5 & \text { Negativa }\end{array}$

10. Estimo que la libre expresión de la cultura de un pueblo o nacionalidad es la única vía para la supervivencia del mismo.

$\begin{array}{lllllll}\text { De acuerdo } & 1 & 2 & 3 & 4 & 5 & \text { En desacuerdo }\end{array}$

11. La diversidad de culturas existentes en el Estado Español, lejos de ser una dificultad para la convivencia entre todos, constituye un patrimonio que hay que conservar y promover.

$\begin{array}{lllllll}\text { De acuerdo } & 1 & 2 & 3 & 4 & 5 & \text { En desacuerdo }\end{array}$

12. Estimo que el ser educado en la propia lengua (castellano, gallego, euskera, valenciano, catalán) y cultura es un derecho fundamental que a nadie puede ser negado.

$\begin{array}{lllllll}\text { De acuerdo } & 1 & 2 & 3 & 4 & 5 & \text { En desacuerdo }\end{array}$

13. La promoción de las tradiciones, costumbres, folklore, etc. de las distintas regiones y nacionalidades del Estado Español es un lujo innecesario y un gasto superfluo.

$\begin{array}{lllllll}\text { De acuerdo } & 1 & 2 & 3 & 4 & 5 & \text { En desacuerdo. }\end{array}$ 


\section{Anexo II}

INTERNAL CONSISTENCY DATA

Split-half correlation $\quad .631$

Spearman-Brown coefficient $\quad .774$

Guttman (Rulon) coefficient $\quad .771$

Coefficient Alpha - All items $\quad .761$

Coefficient Alpha - Odd items $\quad .637$

Coefficient Alpha - Even items $\quad .573$

Aproximate Standard Error of Measurement of Total Score for 15 Z-Score Intervals

\begin{tabular}{lrcrr} 
Z Score & & Total Score & N & Std error \\
\hline$<-3.25$ & $<$ & 3.648 & 0 & - \\
-3.25 TO -2.75 & 3.648 TO & 7.406 & 0 & - \\
-2.75 TO -2.25 & 7.406 TO & 11.165 & 3 & 1.155 \\
-2.25 TO -1.75 & 11.165 TO & 14.923 & 5 & 4.690 \\
-1.75 TO -1.25 & 14.923 TO & 18.682 & 25 & 3.079 \\
-1.25 TO -.75 & 18.682 TO & 22.440 & 31 & 4.476 \\
-.075 TO -.25 & 22.440 TO & 26.198 & 58 & 4.167 \\
.25 TO .25 & 26.198 TO & 29.957 & 49 & 4.118 \\
.25 TO .75 & 29.957 TO & 33.715 & 42 & 4.129 \\
.75 TO 1.25 & 33.715 TO & 37.473 & 43 & 4.162 \\
1.25 TO 1.75 & 37.473 TO & 41.232 & 18 & 3.456 \\
1.75 TO 2.25 & 41.232 TO & 44.990 & 7 & 2.777 \\
2.25 TO 2.75 & 44.990 TO & 48.748 & 2 & 8.246 \\
2.75 TO 3.25 & 48.748 TO & 52.507 & 1 & 4.000 \\
$>=3.25$ & $>=$ & 52.507 & 0 & -
\end{tabular}

Item Reliability Statistics

\begin{tabular}{cccccccc} 
Item & Label & Mean & $\begin{array}{c}\text { Std } \\
\text { Devtn }\end{array}$ & $\begin{array}{c}\text { Total } \\
\text { R }\end{array}$ & $\begin{array}{c}\text { Reliablty } \\
\text { Index }\end{array}$ & $\begin{array}{c}\text { This } \\
\text { R }\end{array}$ & $\begin{array}{c}\text { Item } \\
\text { Alpha }\end{array}$ \\
\hline 1 & P1 & 2.905 & 1.151 & .642 & .740 & .538 & .729 \\
2 & P2 & 2.440 & 1.222 & .500 & .611 & .363 & .748 \\
3 & P3 & 0.648 & 1.076 & .492 & .530 & .375 & .747 \\
4 & P4 & 2.574 & 1.186 & .451 & .535 & .312 & .754 \\
5 & P5 & 2.697 & 1.028 & .413 & .425 & .291 & .755 \\
6 & P6 & 0.616 & 0.922 & .451 & .416 & .345 & .750 \\
7 & P7 & 2.211 & 1.323 & .450 & .595 & .293 & .758 \\
8 & P8 & 2.028 & 1.316 & .640 & .842 & .517 & .730 \\
9 & P9 & 1.246 & 1.182 & .548 & .647 & .423 & .742 \\
10 & P10 & 2.560 & 1.178 & .408 & .481 & .266 & .759 \\
11 & P11 & 2.743 & 1.157 & .648 & .749 & .544 & .728 \\
12 & P12 & 2.873 & 1.103 & .565 & .623 & .452 & .739 \\
13 & P13 & 3.345 & 0.840 & .386 & .324 & .285 & .755
\end{tabular}




\section{Factorial Matrix}

\section{Component Loadings}

$\begin{array}{lrrr} & 1 & 2 & 3 \\ \text { P1 } & 0.682 & 0.073 & 0.174 \\ \text { P2 } & 0.484 & 0.191 & -0.025 \\ \text { P3 } & 0.510 & -0.546 & -0.040 \\ \text { P4 } & 0.432 & 0.185 & 0.441 \\ \text { P5 } & 0.377 & 0.199 & 0.381 \\ \text { P6 } & 0.465 & -0.617 & 0.020 \\ \text { P7 } & 0.396 & 0.071 & 0.383 \\ \text { P8 } & 0.661 & 0.121 & 0.123 \\ \text { P9 } & 0.540 & -0.463 & 0.021 \\ \text { P10 } & 0.366 & -0.018 & -0.722 \\ \text { P11 } & 0.677 & 0.238 & 0.141 \\ \text { P12 } & 0.588 & 0.095 & -0.273 \\ \text { P13 } & 0.390 & 0.512 & -0.149\end{array}$

Variance Explained by Components
1
2
3
3.478
1.357
1.173

Percent of Total Variance Explained

$\begin{array}{lrr}1 & 2 & 3 \\ 26.751 & 10.436 & 9.022\end{array}$

Rotated Loadings

$\begin{array}{lrrr} & 1 & 2 & 3 \\ \text { P1 } & 0.606 & 0.266 & 0.248 \\ \text { P2 } & 0.389 & 0.073 & 0.339 \\ \text { P3 } & 0.127 & 0.728 & 0.120 \\ \text { P4 } & 0.641 & 0.037 & -0.056 \\ \text { P5 } & 0.097 & 0.026 & 0.563 \\ \text { P6 } & 0.107 & 0.764 & 0.026 \\ \text { P7 } & 0.538 & 0.121 & -0.068 \\ \text { P8 } & 0.578 & 0.216 & 0.293 \\ \text { P9 } & 0.216 & 0.668 & 0.116 \\ \text { P10 } & -0.203 & 0.221 & 0.752 \\ \text { P11 } & 0.644 & 0.122 & 0.326 \\ \text { P12 } & 0.272 & 0.216 & 0.555 \\ \text { P13 } & 0.365 & -0.248 & 0.491\end{array}$


Variance Explained by Rotated Components

$\begin{array}{rrr}1 & 2 & 3 \\ 2.299 & 1.872 & 1.836\end{array}$

Percent of Total Variance Explained

$\begin{array}{rrr}1 & 2 & 3 \\ 17.688 & 14.397 & 14.123\end{array}$

